\title{
Towards Automating Structural Discovery in Scanning Transmission Electron Microscopy
}

Nicole Creange ${ }^{1}$, Ondrej Dyck ${ }^{1}$, Christopher Nelson ${ }^{2}$, Rama Vasudevan ${ }^{1}$, Maxim Ziatdinov ${ }^{3}$ and Sergei Kalinin $^{4}$

${ }^{1}$ Oak Ridge National Laboratory, United States, ${ }^{2}$ Oak Ridge National Laboratory, Oak Ridge, Tennessee, United States, ${ }^{3}$ Computational Sciences and Engineering Division, Oak Ridge National Laboratory, Oak Ridge, Tennessee, United States, ${ }^{4}$ Center for Nanophase Materials Sciences, Oak Ridge National Laboratory, Oak Ridge, Tennessee, United States

Over the last decade, electron microscopy has become the mainstay of multiple fields ranging from materials science to condensed matter physics and biology [1-3]. Advancements in resolution, in particular the introduction of aberration correction, has significantly expanded the range of capabilities for uncovering physical phenomenon. However, despite the progression in the development of source and detectors in scanning transmission electron microscopy (STEM), the fundamental principle of scanning microscopies has remained largely the same, namely the sequential acquisition of data and the data collection workflow.

From the information theory viewpoint, the uniform sampling grid corresponds to the case of uniform Bayesian prior, or lack of any prior knowledge of the system[4,5]. This choice is fully justified in the case of the fully unknown sample. However, in most materials system the distribution of data of interest is spatially non-uniform. For example, in ferroelectric thin films, the phenomena of interest occur at interfaces and topological defects, which appear in nonuniform patterns. In nanoparticle systems, phenomena of interest occur at the individual nanoparticles and especially at their junctions, whereas the empty space between them is often featureless. Finally, in atomically-resolved data of systems such as graphene and 2D chalcogenides, phenomena of interest occur at the atomic and extended defects, which can occupy a relatively small fraction of the image plane.

While human operated STEM workflows are the mainstay of the microscopy field, this process is extremely limiting. First and foremost, the latency time of a human operator is well below that of the image data acquisition in STEM. Hence, identification on regions of interest is currently human limited, making studies of dynamic phenomena such as catalysis, growth, or electron-beam manipulation slow. Secondly, the human eye is generally well suited for identification of certain localized features, but the selection is highly biased by the operator experience and interest, introducing a large degree of observation bias in the exploration. Similarly, the discriminative capability of human eye is limited and can be strongly affected by the chosen visualization setting, such as image contrast and color scale. Finally, subtle changes in the image, such as a slight periodicity change, are usually undetected by the human eye and can be revealed only by postexperiment image analysis.

These considerations, have sparked a tremendous interest towards the development of automated experimentation (AE) in general microscopy [6-8] and STEM $[9,10]$ in particular. Some of the specific targets for $\mathrm{AE}$ include the identification and subsequent imaging of specific objects of interest in static systems, aiming at: minimizing the total measurement time and consequent beam damage, imaging dynamic systems, and ultimately controlled and completely automated probe manipulation. In the most general case, implementation of the AE in microscopy requires a set of synergistic instrumental and control advances, including controlled beam motion, rapid analysis of the data to extract descriptors of interest, and feedback based on these descriptors.

Here, we develop various approaches for automated experiment workflows in STEM based on a twotiered approach, including supervised atom identification via deep convolutional neural networks (DCNN) and unsupervised feature of interest discovery via rotationally invariant variational autoencoders (rVAE). The autoencoder output is used as a basis for the acquisition function which guides the Bayesian optimization 
algorithm. This approach is compared with the simpler method based on the linear transform-based AE workflows.

As the first step of the analysis workflow, we use the deep convolutional neural network based semantic segmentation. This choice is dictated by the fact that in most cases of interest in the atomically resolved STEM studies pertain to structures and transformations on the atomic level. Furthermore, DCNN based atom finding is robust and hence can be performed given the pretrained networks for a broad range of image sizes, sampling, and signal to noise ratios. The DCNN predictions can further be improved via ensemble learning and iterative training approaches. It is important to also note that DCNN can be trained for the semantic segmentation of mesoscopic images for determination of specific microstructural elements, e.g. particle and grain boundary finding, determination of the ferroelectric domain walls, etc., allowing for generalization of this approach towards other objects of interest. Subsequently, we implement Bayesian optimization (BO) based on the selected object of interest. We can choose the largest variation in latent space or use human selection. Figure 1 outlines the postulated workflows in which a sub-scan is collected followed by image processing, component selection, and ultimately ending with BO for the determination of where data collection is performed.

These workflows are performed on various datasets including phase separated, 2D, and ferroelectric materials. Each data set presents different difficulties in data collection and location of the data of interest. We show that well-defined large-scale features work in all proposed workflows and as such, the addition of complex algorithms do not enhance the performance of the BO. However, in less well-defined and smaller feature size systems, the use of DCNN and rVAE open avenues for automated features selection and probe manipulation. This work was performed at the Center for Nanophase Materials Sciences, a US Department of Energy Office of Science User Facility.

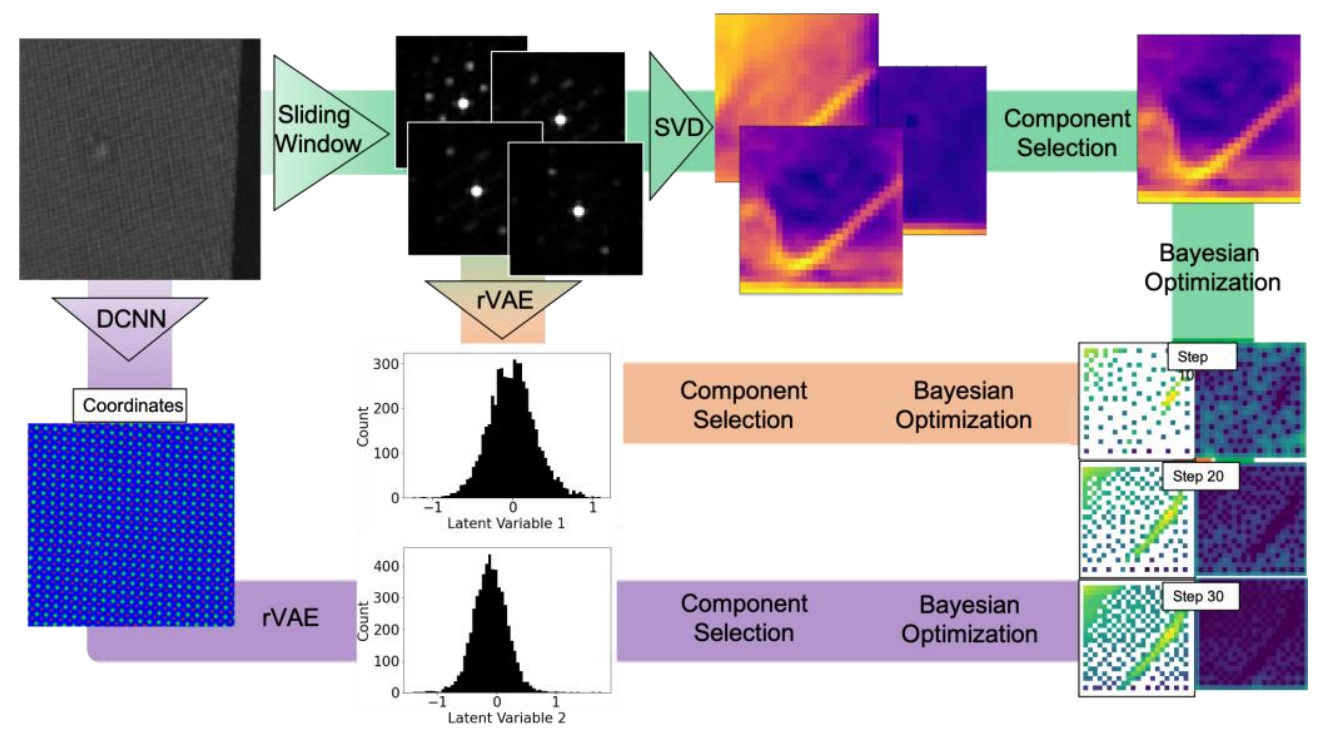

Figure 1. Schematic outlining the various AE workflows including the linear transform-based, supervised, and unsupervised AE workflows.

References 
1. S. J. Pennycook, M. F. Chisholm, A. R. Lupini, M. Varela, K. van Benthem, A. Y. Borisevich, M. P. Oxley, W. Luo and S. T. Pantelides, in Advances in Imaging and Electron Physics, Vol 153, edited by P. W. Hawkes (Elsevier Academic Press Inc, San Diego, 2008), Vol. 153, pp. 327-+.

2. S. J. Pennycook, M. Varela, A. R. Lupini, M. P. Oxley and M. F. Chisholm, Journal of Electron Microscopy 58 (3), 87-97 (2009).

3. Y. Jiang, Z. Chen, Y. M. Hang, P. Deb, H. Gao, S. E. Xie, P. Purohit, M. W. Tate, J. Park, S. M. Gruner, V. Elser and D. A. Muller, Nature 559 (7714), 343-+ (2018).

4. B. Lambert, A Student's Guide to Bayesian Statistics. (SAGE Publications Ltd; 1 edition, 2018).

5. O. Martin, Bayesian Analysis with Python: Introduction to statistical modeling and probabilistic programming using PyMC3 and ArviZ, 2nd Edition. (Packt Publishing, 2018).

6. S. V. Kalinin, E. Strelcov, A. Belianinov, S. Somnath, R. K. Vasudevan, E. J. Lingerfelt, R. K. Archibald, C. M. Chen, R. Proksch, N. Laanait and S. Jesse, ACS Nano 10 (10), 9068-9086 (2016).

7. P. Zahl, T. Wagner, R. Moller and A. Klust, Journal of Vacuum Science \& Technology B 28 (3), C4E39 (2010).

8. A. Krull, P. Hirsch, C. Rother, A. Schiffrin and C. Krull, Commun. Phys. 3 (1), 8 (2020).

9. S. V. Kalinin, A. Borisevich and S. Jesse, Nature 539 (7630), 485-487 (2016).

10. O. Dyck, S. Jesse and S. V. Kalinin, MRS Bull. 44 (9), 669-670 (2019). 